\title{
Inverted Internal Limiting Membrane Flap Technique versus Internal Limiting Membrane Peeling for Large Macular Holes: A Meta-Analysis of Randomized Controlled Trials
}

\author{
Ji-Guo Yu Jing Wang Yi Xiang \\ Department of Ophthalmology, The Central Hospital of Wuhan, Tongji Medical College, Huazhong University of \\ Science and Technology, Wuhan, China
}

\section{Keywords}

Inverted internal limiting membrane flap technique . Internal limiting membrane peeling - Large macular hole . Meta-analysis

\begin{abstract}
Background: Vitrectomy with internal limiting membrane (ILM) peeling is an effective surgical procedure for the treatment of macular holes (MHs). However, there is a possibility of poor postoperative anatomical closure with conventional ILM peeling for MHs larger than $400 \mu \mathrm{m}$. Therefore, a novel inverted ILM flap technique was developed for such cases. Objectives: This meta-analysis study was performed to evaluate and compare the anatomical and visual outcomes of the inverted ILM flap technique and ILM peeling in large MHs. Methods: The Cochrane Library, PubMed, and Embase databases were searched to identify randomized controlled trials (RCTs). The trial eligibility and risk of bias were assessed according to Cochrane review methods. The primary outcome measures included $\mathrm{MH}$ closure rate and postoperative visual acuity (VA). Subgroup analysis of postoperative VA based on follow-up time was also conducted. Pooled odds ratios (ORs), weighted mean difference (WMD), and 95\% confidence intervals $(\mathrm{Cls})$ were calculated. Statistical analysis was performed using RevMan 5.3 software. Results: Five
\end{abstract}

RCTs with a total of 155 eyes in the inverted ILM flap group and 161 eyes in the ILM peeling group were included in this meta-analysis. Statistical meta-analysis revealed that the overall $\mathrm{MH}$ closure rate in the inverted ILM flap group was significantly higher than that in the ILM peeling group (OR= $3.10 ; 95 \% \mathrm{Cl}: 1.25-7.66 ; p=0.01)$. The postoperative $\mathrm{VA}$ was significantly better in the inverted ILM flap group than the ILM peeling group (WMD $=-0.14 ; 95 \% \mathrm{Cl}:-0.21$ to $-0.07 ; p$ $=0.0002$ ). The subgroup meta-analysis indicated that the postoperative VA was significantly better in the inverted ILM flap group than the ILM peeling group (WMD $=-0.17 ; 95 \%$ Cl: -0.26 to $-0.08 ; p=0.0004$ ) at the 3 -month follow-up. However, no significant difference was observed between the 2 groups at the 6-month follow-up (WMD $=-0.09 ; 95 \%$ $\mathrm{Cl}:-0.20$ to $0.02 ; p=0.10)$. Conclusions: Vitrectomy with the inverted ILM flap technique showed a higher anatomical closure rate as well as visual gain - although only in the short term as no difference in visual recovery was found at the 6-month follow-up - than did ILM peeling in large MHs. The inverted ILM flap technique should be considered as a preferred and routine procedure for the treatment of patients with MHs larger than $400 \mu \mathrm{m}$.

(c) 2021 The Author(s). Published by S. Karger AG, Basel

Ji-Guo Yu and Jing Wang contributed equally to this work. karger@karger.com www.karger.com/ore

Karger $\stackrel{\text { ' }}{5}$

GOPEN ACCESS
(C) 2021 The Author(s)

Published by S. Karger AG, Basel

This is an Open Access article licensed under the Creative Commons Attribution-NonCommercial-4.0 International License (CC BY-NC) (http://www.karger.com/Services/OpenAccessLicense), applicable to the online version of the article only. Usage and distribution for commercial purposes requires written permission.
Correspondence to:

Yi Xiang, xyyanke@163.com 


\section{Introduction}

Macular holes (MHs) are anatomical defects in the fovea of retina characterized by the interruption of neural retinal layers from the internal limiting membrane (ILM) to the photoreceptor layer. This can cause blurred and distorted central vision and occurs more frequently in women over the age of 50 years [1,2]. Vitrectomy was first described by Kelly and Wendel [3] and is considered the most effective surgical procedure for the treatment of MHs. Although internal limiting membrane (ILM) peeling is performed during surgery, the anatomical success rate of surgery is not consistent for different sizes of MHs. The size of $\mathrm{MH}$ affects the healing rate. Previous studies reported that the success rate of $\mathrm{MH}$ surgery is $91-98 \%$ for MHs $<400 \mu \mathrm{m}[4-6]$. However, the anatomical success rate of MHs larger than $400 \mu \mathrm{m}$ can be as low as $50-80 \%$ [7-9]. Therefore, a novel inverted ILM flap technique was developed for the treatment of large MHs [10].

Although studies suggest that the inverted ILM flap technique achieves better anatomical and visual outcomes compared to ILM peeling [11-13], some studies have shown that the outcomes of the inverted ILM flap technique in large MHs is statistically similar to that observed in conventional ILM peeling $[8,14-16]$. At present, a metaanalysis using randomized controlled trials (RCTs) to compare anatomical and visual outcomes between the 2 methods in MHs larger than $400 \mu \mathrm{m}$ has not been published. Therefore, we performed a meta-analysis of RCTs to evaluate the efficacy of vitrectomy with the inverted ILM flap technique to determine if it has high closure rates and good improvements in visual acuity (VA) for large MHs.

\section{Methods}

\section{Search Strategy}

This meta-analysis was conducted in accordance with the Cochrane Handbook for Systematic Reviews of Interventions and Preferred Reporting Items for Systematic Reviews and Meta-Analysis (PRISMA) Statement. The PRISMA 2009 Checklist is presented in online suppl. Material 1 (for all online suppl. material, see www.karger.com/doi/10.1159/000515283). An electronic search of PubMed, Embase, and Cochrane Library until June 7, 2020, was performed to identify relevant studies. The following terms were used for the searches: "macular hole," "inverted internal limiting membrane flap," "inverted ILM flap," "inverted flap," "internal limiting membrane peeling," "ILM peeling," and "standard ILM peel." The literature search results were imported into a reference management software (EndNote X4; Thomson Reuters, New York, NY, USA), and duplicate reports were deleted. Afterward, 2 evaluators (Y.X. and J.-G.Y.) read the titles and abstracts to eliminate irrelevant reports. Subsequently, the remaining reports were reviewed by reading the full text to determine if they met the inclusion criteria. In addition, their reference lists were also searched for additional articles.

\section{Inclusion and Exclusion Criteria}

The studies were included if they met the following criteria: (1) studies must be RCTs, (2) studies must compare inverted ILM flap technique and ILM peeling, (3) studies should include patients with MHs larger than $400 \mu \mathrm{m}$, (4) studies should report the anatomical hole closure rate and VA, and (5) the follow-up duration must be at least 3 months or longer. Exclusion criteria were as follows: (1) studies without the ILM peeling group; (2) studies that compared with other surgeries, such as modified inverted ILM flap techniques; (3) the data could not be used for meta-analysis; (4) studies that included patients with macular retinoschisis or MHs with retinal detachment; and (5) retrospective studies, case reports, and review articles.

\section{Data Extraction}

Two reviewers (J.-G.Y. and J.W.) independently extracted the following demographic and clinical data from each of the study: first author, year of publication, study location, study design, number of eyes, mean age, sex, minimum diameter, hole closure rate, and follow-up period. Any discrepancies in data abstraction were resolved via discussion with another author (Y.X.).

\section{Quality Assessment}

The quality of the RCTs included was assessed to identify any potential risk of bias using the Cochrane Risk of Bias assessment tool. Seven domains were assessed: (1) random sequence generation, (2) allocation concealment, (3) blinding of participants and personnel, (4) blinding of outcome assessment, (5) incomplete outcome data, (6) selective reporting, and (7) other bias. Each domain was classified into "low risk of bias," "high risk of bias," and "unclear risk of bias." Three reviewers (J.-G.Y., J.W., and Y.X.) independently evaluated the clinical trials using this tool, and discrepancies were resolved via discussion.

\section{Statistical Analysis}

Statistical analysis was performed using RevMan software (version 5.3; Cochrane Collaboration, Oxford, UK). For continuous outcomes, the means and standard deviations (SDs) were used to calculate the weighted mean difference (WMD) with $95 \%$ confidence intervals (CI). However, odds ratios (ORs) with 95\% CI were calculated for dichotomous outcomes. Statistical heterogeneity between studies was assessed using the $\chi^{2}$ test, with $p<0.05$ and $I^{2}$ $>50 \%$ indicating significant heterogeneity [17]. Heterogeneity was considered low when $I^{2} \leq 50 \%$, and the data were analyzed using the fixed-effects model. Instead, the random-effects model was used when $I^{2}>50 \%$ [18]. Potential publication bias was examined via visual inspection of a funnel plot [19]. A $p$ value $<0.05$ was considered statistically significant.

\section{Results}

\section{Study Selection}

A total of 914 records were identified through a systematic literature search. There were 103 records left after 


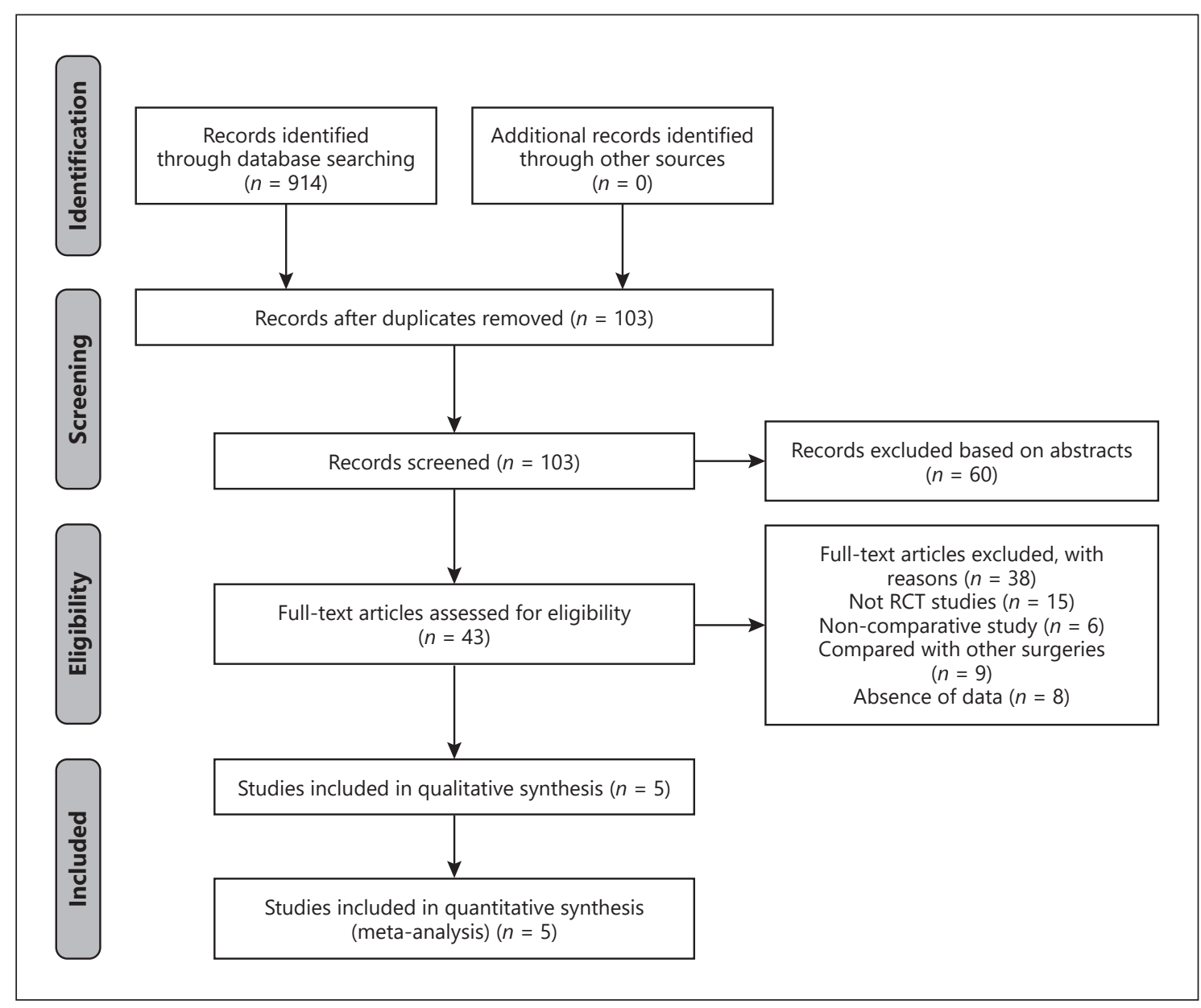

Fig. 1. Flowchart of the literature search and selection. RCT, randomized controlled trial.

the duplicates were removed, of which 60 reports were excluded based on the title and abstract. The remaining 43 studies were assessed by reading the full text. Out of the 43 reports, 15 studies were not RCTs, 6 studies were noncomparative studies, 9 studies made comparisons with other surgeries, and 8 studies lacked relevant data. Therefore, these reports were excluded from the metaanalysis. The 5 RCTs that met our inclusion criteria were included in the final meta-analysis $[10,11,14-16]$. The study selection process is shown in Figure 1.

\section{Characteristics of the Studies}

The selected studies included a total of 316 eyes, 155 of which were in the inverted ILM flap group and 161 in the ILM peeling group. Two studies were performed in India $[11,14]$, and one each were performed in Italy [16], Mexico [15], and Poland [10]. All of the studies were pub- lished between 2010 and 2018. The age of the patients ranged from 60 to 71 years. The number of eyes examined in the studies ranged from 12 to 51 . The characteristics of each study are shown in Table 1 , and the risk of bias assessment is shown in Figure 2. Overall, the included studies were at low risk for bias.

\section{Outcomes of Meta-Analysis}

Overall MH Closure Rate

The overall $\mathrm{MH}$ closure rate was compared between the inverted ILM flap and ILM peeling group across 5 studies. No statistical heterogeneity was found $\left(I^{2}=0 \%\right)$. Therefore, the data were analyzed using the fixed-effects model. The pooled data showed that the $\mathrm{MH}$ closure rate in the inverted ILM flap group was significantly higher than that in the ILM peeling group $(\mathrm{OR}=3.10 ; 95 \% \mathrm{CI}$ : $1.25-7.66 ; p=0.01$ ) (shown in Fig. 3). 
Table 1. Summary of the characteristics of the included studies

\begin{tabular}{|c|c|c|c|c|c|c|c|c|c|c|c|c|c|}
\hline \multirow[t]{2}{*}{ Author } & \multirow[t]{2}{*}{$\begin{array}{l}\text { Study } \\
\text { location }\end{array}$} & \multirow[t]{2}{*}{$\begin{array}{l}\text { Study } \\
\text { design }\end{array}$} & \multicolumn{2}{|c|}{ Eyes, $n$} & \multicolumn{2}{|c|}{ Mean age, years } & \multicolumn{2}{|c|}{$\begin{array}{l}\text { Gender (male/ } \\
\text { female) }\end{array}$} & \multicolumn{2}{|c|}{$\begin{array}{l}\text { Minimum } \\
\text { diameter }\end{array}$} & \multicolumn{2}{|c|}{$\begin{array}{l}\text { Hole closure } \\
\text { rate, \% }\end{array}$} & \multirow{2}{*}{$\begin{array}{l}\text { Follow- } \\
\text { up, } \\
\text { months }\end{array}$} \\
\hline & & & $\begin{array}{l}\text { ILM } \\
\text { flap }\end{array}$ & $\begin{array}{l}\text { ILM } \\
\text { peeling }\end{array}$ & $\begin{array}{l}\text { ILM } \\
\text { flap }\end{array}$ & $\begin{array}{l}\text { ILM } \\
\text { peeling }\end{array}$ & $\begin{array}{l}\text { ILM } \\
\text { flap }\end{array}$ & $\begin{array}{l}\text { ILM } \\
\text { peeling }\end{array}$ & $\begin{array}{l}\text { ILM } \\
\text { flap }\end{array}$ & $\begin{array}{l}\text { ILM } \\
\text { peeling }\end{array}$ & $\begin{array}{l}\text { ILM } \\
\text { flap }\end{array}$ & $\begin{array}{l}\text { ILM } \\
\text { peeling }\end{array}$ & \\
\hline Kannan et al. [14] & India & RCT & 30 & 30 & 59.37 & 61.17 & $11 / 19$ & $17 / 13$ & 803.33 & 759.97 & 90 & 76.7 & 6 \\
\hline Iovino et al. [16] & Italy & RCT & 20 & 20 & 71 & 69 & $9 / 11$ & $7 / 13$ & 666.95 & 664.6 & 100 & 100 & 6 \\
\hline Michalewska et al. [10] & Poland & RCT & 50 & 51 & 66 & 65 & $13 / 33$ & $8 / 32$ & 759 & 698 & 98 & 88 & 12 \\
\hline
\end{tabular}

RCT, randomized controlled trial; ILM, internal limiting membrane; nr, not reported.

Fig. 2. Assessment of the risk of bias in included studies. Risk of bias graph: judgments about each "risk of bias" item presented as percentages across all included studies (a); risk of bias summary: the detailed risk of bias values for each article (b). + , low risk of bias; -, high risk of bias; ?, unclear risk of bias.

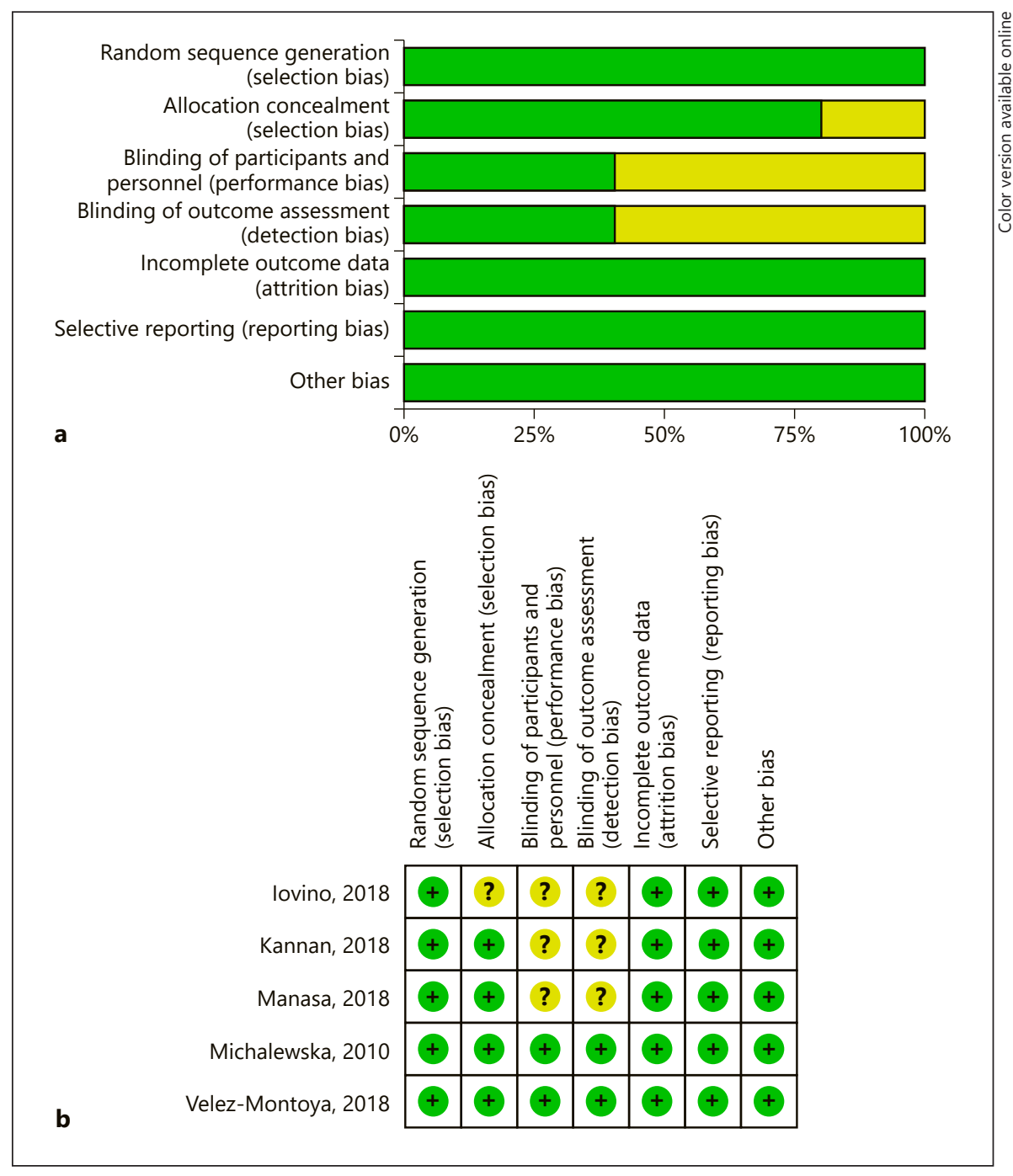




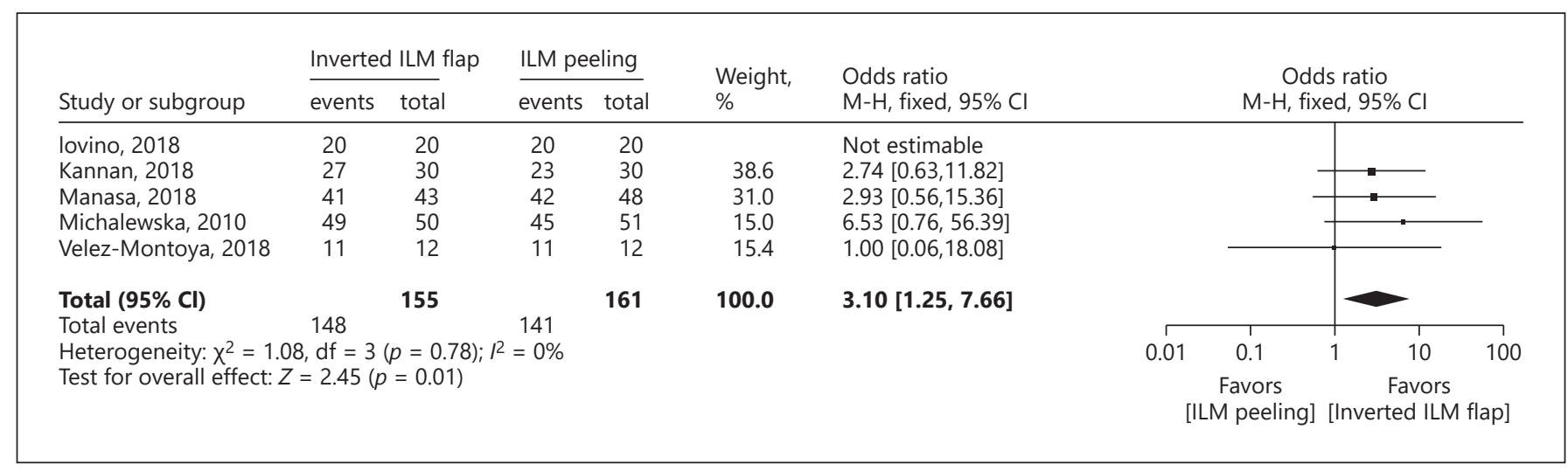

Fig. 3. Forest plots of macular hole closure rate between inverted ILM flap and ILM peeling groups. ILM, internal limiting membrane.

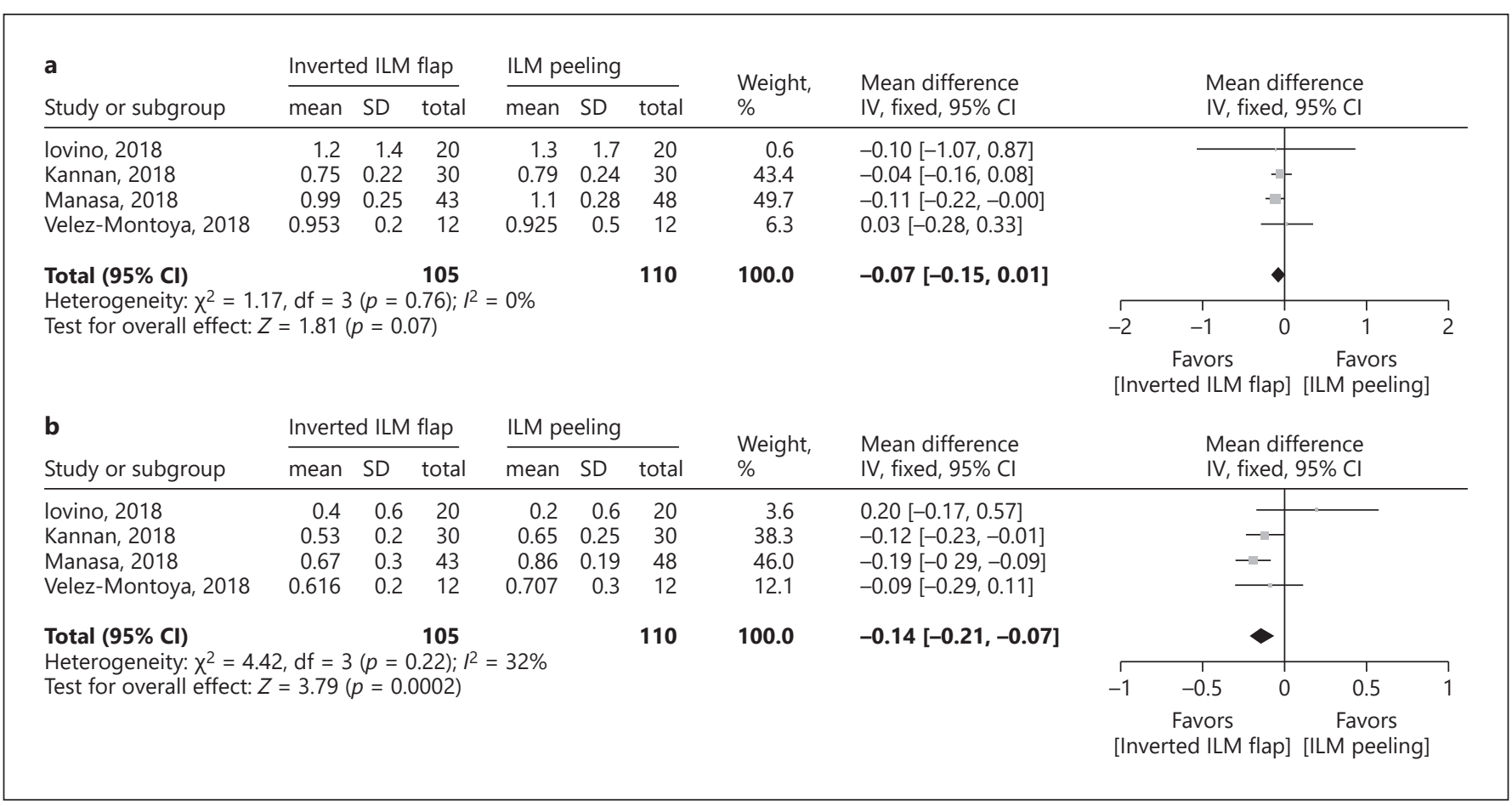

Fig. 4. Forest plots of visual acuity (logMAR units) between inverted ILM flap and ILM peeling groups. Preoperative visual acuity (a); postoperative visual acuity (b). ILM, internal limiting membrane.

Preoperative Visual Acuity

There were only 4 studies pooled together to compare the preoperative VA (logMAR units) between 2 groups because one study [10] did not report the SD of the preoperative VA. No statistical heterogeneity was found $\left(I^{2}=0 \%\right)$, and the data were analyzed using the fixed-ef- fects model. The forest plots revealed that the difference in the preoperative VA was not statistically significant between the inverted ILM flap group and the ILM peeling group (WMD $=-0.07 ; 95 \% \mathrm{CI}:-0.15$ to $0.01 ; p=0.07$ ) (shown in Fig. 4a). 


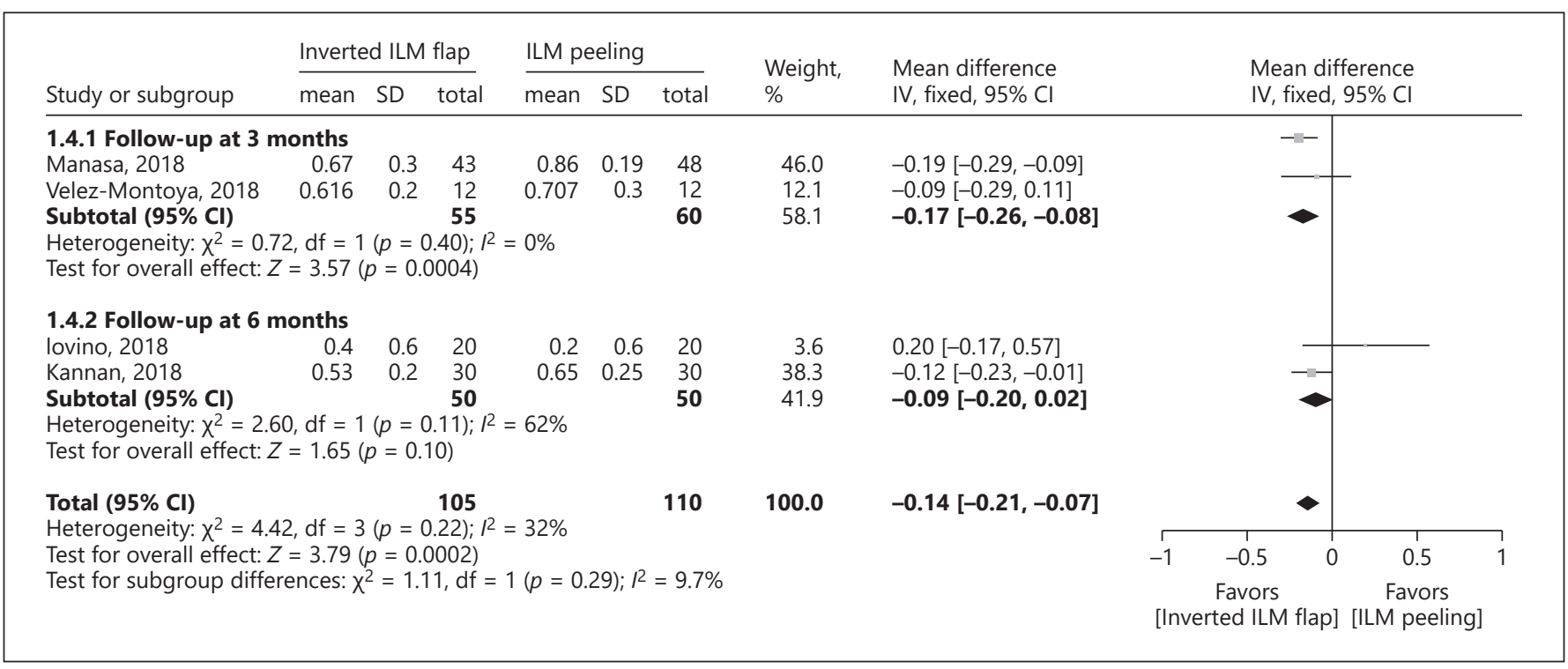

Fig. 5. Forest plots of postoperative visual acuity (logMAR units) based on the follow-up duration in the subgroup analysis. ILM, internal limiting membrane.

\section{Postoperative Visual Acuity}

Four studies were pooled together to compare the postoperative VA (logMAR units) between the 2 groups. No statistical heterogeneity was found $\left(I^{2}=32 \%\right)$; hence, the data were also analyzed using the fixed-effects model. Meta-analysis of these data showed that the postoperative VA was significantly better in the inverted ILM flap group than the ILM peeling group (WMD $=-0.14 ; 95 \% \mathrm{CI}$ : -0.21 to $-0.07 ; p=0.0002$ ) (shown in Fig. $4 b$ ).

The subgroup analysis of postoperative VA was performed based on the follow-up duration. The follow-up duration was divided into 3 and 6 months. In the subgroup analysis of the follow-up duration at 3 months, 2 studies were pooled together which included 55 eyes from the inverted ILM flap group and 60 eyes from the ILM peeling group. No significant heterogeneity $\left(I^{2}=0 \%\right)$ was found across the studies; thus, the data were analyzed through the fixed-effects model. Meta-analysis of these data showed that the postoperative VA at 3 months was significantly better in the inverted ILM flap group than the ILM peeling group (WMD $=-0.17 ; 95 \% \mathrm{CI}:-0.26$ to $-0.08 ; p=0.0004$ ) (shown in Fig. 5). In the subgroup analysis of the follow-up duration at 6 months, 2 studies were pooled together, which included 105 eyes from the inverted ILM flap group and 110 eyes from the ILM peeling group. The pooled results did not show any significant difference between the 2 groups at 6 months (WMD = -0.09 ; $95 \%$ CI: -0.20 to $0.02 ; p=0.10$ ) (shown in Fig. 5).
Publication Bias Analysis

The funnel plots showed that the distribution of the plots was symmetric. Most points were distributed in the middle and top of the baseline and were located in the range of the inverted funnel, which indicated that no serious publication bias affected the credibility of this study (shown in Fig. 6).

\section{Discussion}

Previous studies reported that vitrectomy with ILM peeling can remove the anteroposterior and tangential tractional forces of the macular retina surface to promote the healing of the hole [20-22]. For MHs smaller than 400 $\mu \mathrm{m}$, ILM peeling was enough to achieve a high successful anatomic healing rate [23]. However, for MHs larger than $400 \mu \mathrm{m}$, conventional ILM peeling was not enough to ensure the successful healing of the hole $[24,25]$. This could be due to the distance between the broken ends of the retina that contains large neural defects which are difficult to fill with the proliferation of glial cells alone [14] unless a scaffold was provided for the migration of glial cells. Thus, a novel inverted ILM flap technique was developed for the treatment of large MHs. The current meta-analysis of 5 RCTs showed that the overall MH closure rate of the inverted ILM flap group was significantly higher than that in the ILM peeling group. This suggests that 


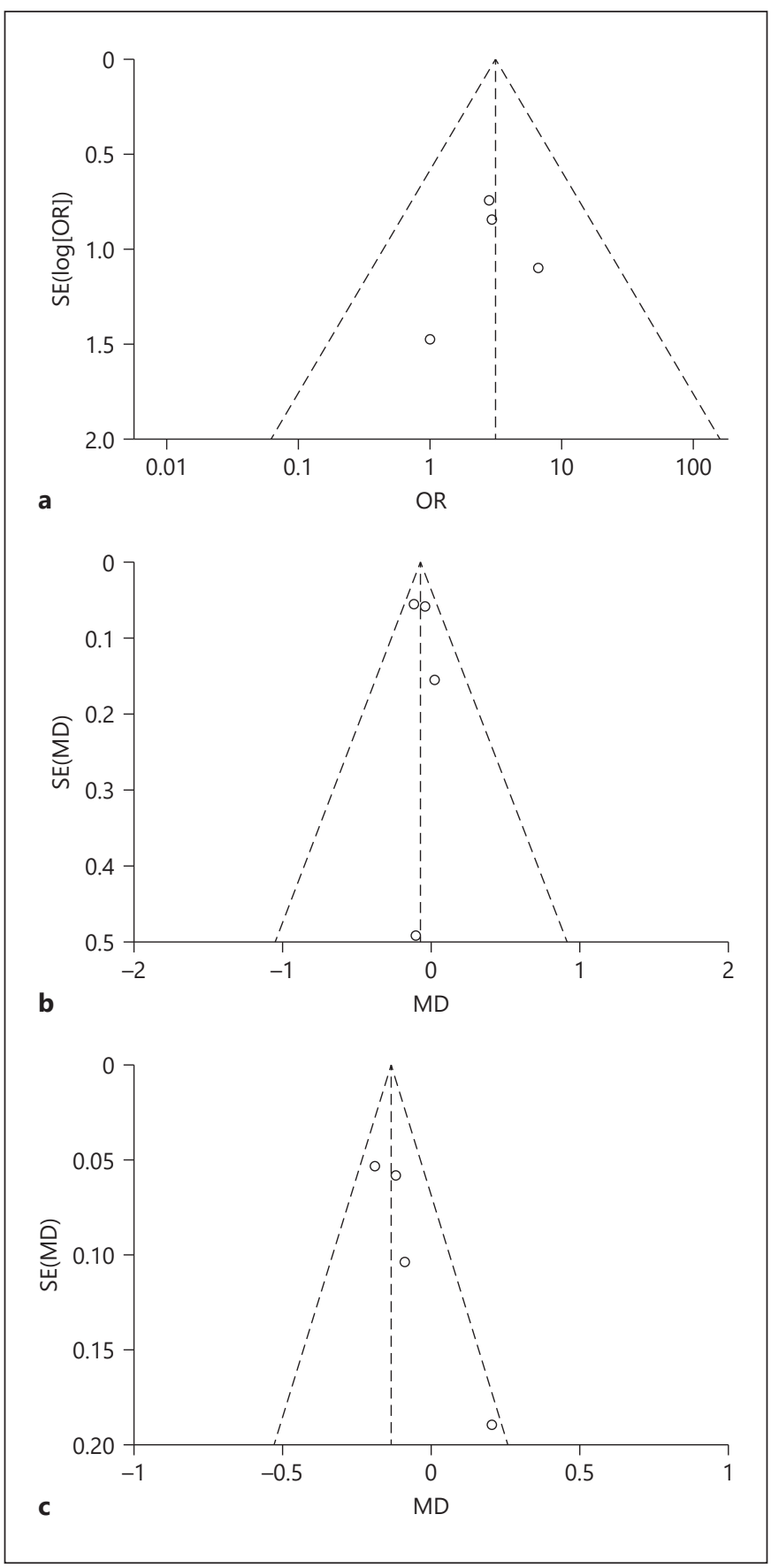

Fig. 6. Funnel plots for evaluating the publication bias. Macular hole closure rate (a); preoperative visual acuity (b); postoperative visual acuity $(\mathbf{c})$.

the inverted ILM flap technique should be considered first and used more often for the treatment of large MHs.

The inverted ILM flap technique provides a smooth and gap-free natural scaffold for the proliferation and mi- gration of glial cells $[10,26]$. The Muller cells were also activated to secrete neurotrophic factors and basic fibroblast growth factors on the surface of the ILM, which may promote the survival of the retinal neurons and help the photoreceptor cells grow to the center of the fovea [27]. Manasa et al. [11] reported that the ILM flap not only provides a basement membrane for cells to proliferate but also provides an environment for the photoreceptors to align to a new position in direct proximity to the fovea. Moreover, the ILM flap on MHs can create a closed compartment that enables the retinal pigment epithelium to pump out the subretinal fluid effectively. It prevents further leakage of the fluid into the fovea, thereby keeping the hole dry and contributing to $\mathrm{MH}$ closure [11]. This may explain the higher anatomical closure rate in the inverted ILM flap group than in the ILM peeling group.

Improved functional outcomes after surgical closures of large MHs may depend on the restoration of the outer retinal layers. $\mathrm{MH}$ surgery is expected to result in a type 1 anatomical closure. Type 1 closure is considered the best healing of $\mathrm{MH}$ with improved visual function recovery, smaller photoreceptor layer defects, and normal retinal thickness at the end of the follow-up [28]. In previous studies, the inverted ILM flap was reported to have a higher incidence of type 1 closure in large $\mathrm{MHs}$ compared to the standard ILM peeling [11]. Thus, the functional outcome should be significantly better in the inverted ILM flap group. This was also consistent with the results of our meta-analysis, which showed that the postoperative VA was significantly better in the inverted ILM flap group than the ILM peeling group. There was no significant difference in the preoperative VA between the 2 groups, which could have reduced the preintervention effects to postoperative VA.

The subgroup analysis of postoperative VA was based on the follow-up duration to determine the short- and long-term visual efficacy. The results showed that the patients on the inverted flap technique had a significant visual improvement at the 3-month follow-up; however, there was no significant difference between the 2 groups at the 6-month follow-up. It is possible that patients with the inverted ILM flap technique had faster visual recovery during the short-term follow-up of 3 months, which depended on its high $\mathrm{MH}$ closure rate. On the other hand, the best-corrected visual acuity in the ILM peeling group continued to show signs of improvement up to 6 months after surgery. During the following months, the following $\mathrm{MH}$ healing processes were observed: a proliferation of Muller cells, regeneration of retinal tissue starting from the external limiting membrane, photoreceptor migra- 
tion into the retina defect, and reposition, followed by restoration of the ellipsoid-zone layer [29-31]. For as long as the $\mathrm{MH}$ healed, the possibility of improved functional outcomes can be obtained.

The inverted ILM flap technique was beneficial to the $\mathrm{MH}$ healing; however, after the MH closure, no statistically significant difference in the improvement of the postoperative VA was found upon long-term follow-up regardless of the surgical method used. In addition, it should be understood that the size of the $\mathrm{MH}$ affects postoperative MH healing and vision recovery. Furthermore, even with $\mathrm{MH}$ closure, the foveal structure did not return to the normal-layered structure of the retina in large $\mathrm{MHs}$ $[24,32]$. Therefore, no matter which surgical method is used, the visual recovery after vitrectomy is limited at a longer follow-up.

In general, larger $\mathrm{MH}$ apertures correlate with worse preoperative VA. When the aperture of the $\mathrm{MH}$ is $>600$ $\mu \mathrm{m}$, or even $>1,000 \mu \mathrm{m}$, a large number of photoreceptor cells were apoptotic and damaged. Ooto et al. [33] suggested that the foveal photoreceptor layer in the $\mathrm{MH}$ might be destroyed and had irreversible damage. When an $\mathrm{MH}$ heals after vitrectomy, the photoreceptor inner segment and outer segment (IS/OS) layer and external limiting membranes would return gradually after surgery, but the normal-layered structure of the outer retina was not restored completely in all cases. Ota et al. [34] demonstrated that the fovea loses its original stratified structure in many cases after a large $\mathrm{MH}$ heals. Moreover, preoperative $\mathrm{MH}$ apertures may be related to the restoration of the outer retina. No further subgroup comparison of visual recovery for different apertures of large MHs was performed in the included literature. During the longterm follow-up, we found that there was no statistically significant difference in visual recovery between the inverted ILM flap group and the ILM peeling group. While the inverted ILM flap technique has advantages on the $\mathrm{MH}$ closure rate, it did not result in better visual recovery in the long-term follow-up. Because the key to the prognosis of vision is the restoration and rearrangement of photoreceptor cells, this process is still difficult for large MHs, regardless of the surgical technique that is used. Although there was no statistically significant difference between the 2 groups at the 6 -month follow-up, the forest plot showed that inverted ILM flap tended to result in a better visual prognosis than the ILM peeling group.

All of the 5 studies included in this study excluded highly myopic MHs. People with high myopia have poor VA, which is worsened by an $\mathrm{MH}$ and can even result in $\mathrm{MH}$ retinal detachment. Although surgical techniques such as
ILM peeling and inverted ILM flap are also applied to highly myopic MHs, there is a high probability that the visual prognosis is lower than that of patients with nonhigh myopic MHs. A comparison of the closure rate and visual recovery after surgery between highly and nonhigh myopic MHs was not performed in this study due to a lack of relevant data. Although both of them are classified as a refractory $\mathrm{MH}$, we believe that these 2 types of holes are different regarding pathogenesis, operation risk, closure rate, and visual prognosis. Therefore, it is not appropriate to compare them together, but it is possible to compare the ILM peeling and inverted ILM flap techniques for large MHs with high myopia alone. Although previous studies have found that the inverted flap technique had better efficacy for patients with highly myopic MHs regarding closure rate, foveal microstructure, and postoperative best-corrected visual acuity 6 months after surgery $[35,36]$, it would be more convincing if the same conclusion can be drawn from a systematic review and meta-analysis of related RCTs, which will be addressed in future studies.

There were some limitations in this study. First, all of the included studies had small sample sizes. Second, follow-up durations in the included studies were not long enough for better observation of VA recovery. Third, the sizes of MHs may affect the closure rate and functional recovery. The closure type may also affect the postoperative VA. Unfortunately, we were not able to perform a subgroup analysis for these variables due to the lack of data. Last, the area of ILM peeling, toxicity of the ILM stain, air or longer-acting gas tamponade, postoperative face-down position, and time may also have influenced the closure rates. However, we were unable to find sufficient data to investigate these parameters. Therefore, more RCTs with larger sample sizes and longer follow-up durations for evaluating the aforementioned parameters are required.

\section{Conclusion}

This meta-analysis provides evidence that vitrectomy with the inverted ILM flap technique had a higher anatomical closure rate than ILM peeling in large MHs. The ILM flap technique had a better visual recovery in the short-term follow-up, though the advantage of visual recovery was not found at a longer follow-up. The inverted ILM flap technique should be considered as a preferred and routine procedure for the treatment of patients with larger MHs. However, more RCTs with larger sample sizes and longer follow-up durations are recommended to confirm the efficacy of the inverted ILM flap technique. 


\section{Statement of Ethics}

The research was conducted ethically in accordance with the World Medical Association Declaration of Helsinki. Our research did not include original overview of clinical trials, and this article is a summary analysis of published data. Therefore, ethical approval was not required.

\section{Conflict of Interest Statement}

The authors have no conflicts of interest to declare.

\section{Funding Sources}

No funding.

\section{References}

1 Morgan CM, Schatz H. Idiopathic macular holes. Am J Ophthalmol. 1985 Apr 15;99(4): 437-44.

2 Mann DF. Idiopathic macular hole. Optom Clin. 1996;5(1):95-110.

3 Kelly NE, Wendel RT. Vitreous surgery for idiopathic macular holes. Results of a pilot study. Arch Ophthalmol. 1991 May;109(5): 654-9.

4 Lai MM, Williams GA. Anatomical and visual outcomes of idiopathic macular hole surgery with internal limiting membrane removal using low-concentration indocyanine green. Retina. 2007 Apr-May;27(4):477-82.

5 Park DW, Sipperley JO, Sneed SR, Dugel PU, Jacobsen J. Macular hole surgery with internal-limiting membrane peeling and intravitreous air. Ophthalmology. $1999 \mathrm{Jul} ; 106(7)$ : 1392-8; discussion 97-8.

6 Wolf S, Reichel MB, Wiedemann P, Schnurrbusch UEK. Clinical findings in macular hole surgery with indocyanine green-assisted peeling of the internal limiting membrane. Graefes Arch Clin Exp Ophthalmol. 2003 Jul; 241(7):589-92.

7 Ip MS, Baker BJ, Duker JS, Reichel E, Baumal CR, Gangnon R, et al. Anatomical outcomes of surgery for idiopathic macular hole as determined by optical coherence tomography. Arch Ophthalmol. 2002 Jan;120(1):29-35.

8 Narayanan R, Singh SR, Taylor S, Berrocal $\mathrm{MH}$, Chhablani J, Tyagi M, et al. Surgical outcomes after inverted internal limiting membrane flap versus conventional peeling for very large macular holes. Retina. 2019 Aug; 39(8):1465-9.

9 Rizzo S, Tartaro R, Barca F, Caporossi T, Bacherini D, Giansanti F. Internal limiting membrane peeling versus inverted flap technique for treatment of full-thickness macular holes: a comparative study in a large series of patients. Retina. 2018 Sep;38(Suppl 1):S73-8.

\section{Author Contributions}

All authors contributed to the study conception and design. Literature search and screening, data collection and analysis, generation of the figures, data interpretation, and preparation of the manuscript were performed by Ji-Guo Yu and Jing Wang. Study design, study analysis, writing of the discussion, and revision of the manuscript were performed by Yi Xiang. All authors read and approved the final version of the manuscript.

\section{Data Availability Statement}

All data generated or analyzed during this study are included in this article. Further enquiries can be directed to the corresponding author.
10 Michalewska Z, Michalewski J, Adelman RA, Nawrocki J. Inverted internal limiting membrane flap technique for large macular holes. Ophthalmology. 2010;117(10):2018-25.

11 Manasa S, Kakkar P, Kumar A, Chandra P, Kumar V, Ravani R. Comparative evaluation of standard ILM peel with inverted ILM flap technique in large macular holes: a prospective, randomized study. Ophthalmic Surg Lasers Imaging Retina. 2018 Apr 1;49(4):23640.

12 Pak KY, Park JY, Park SW, Byon IS, Lee JE. Efficacy of the perfluoro-n-octane-assisted single-layered inverted internal limiting membrane flap technique for large macular holes. Ophthalmologica. 2017;238(3):133-8.

13 Yamashita T, Sakamoto T, Terasaki H, Iwasaki M, Ogushi Y, Okamoto F, et al. Best surgical technique and outcomes for large macular holes: retrospective multicentre study in Japan. Acta Ophthalmol. 2018 Dec;96(8): e904-10.

14 Kannan NB, Kohli P, Parida H, Adenuga OO, Ramasamy K. Comparative study of inverted internal limiting membrane (ILM) flap and ILM peeling technique in large macular holes: a randomized-control trial. BMC Ophthalmol. 2018 Jul 20;18(1):177.

15 Velez-Montoya R, Ramirez-Estudillo JA, Sjoholm-Gomez de Liano C, Bejar-Cornejo F, Sanchez-Ramos J, Guerrero-Naranjo JL, et al. Inverted ILM flap, free ILM flap and conventional ILM peeling for large macular holes. Int J Retina Vitreous. 2018;4:8.

16 Iovino C, Caminiti G, Miccoli M, Nasini F, Casini G, Peiretti E. Comparison of inverted flap and subretinal aspiration technique in full-thickness macular hole surgery: a randomized controlled study. Eur J Ophthalmol. 2018 May;28(3):324-8
17 Higgins JP, Thompson SG, Deeks JJ, Altman DG. Measuring inconsistency in meta-analyses. BMJ. 2003 Sep 6;327(7414):557-60.

18 DerSimonian R, Kacker R. Random-effects model for meta-analysis of clinical trials: an update. Contemp Clin Trials. 2007 Feb;28(2): 105-14.

19 Stuck AE, Rubenstein LZ, Wieland D. Bias in meta-analysis detected by a simple, graphical test. Asymmetry detected in funnel plot was probably due to true heterogeneity. BMJ. 1998 Feb 7;316(7129):469; author reply 70-1.

20 Smiddy WE, Flynn HW Jr. Pathogenesis of macular holes and therapeutic implications. Am J Ophthalmol. 2004 Mar;137(3):525-37.

21 Hisatomi T, Enaida H, Sakamoto T, Kanemaru T, Kagimoto T, Yamanaka I, et al. Cellular migration associated with macular hole: a new method for comprehensive bird's-eye analysis of the internal limiting membrane. Arch Ophthalmol. 2006 Jul;124(7):1005-11.

22 Ehlers JP, Xu D, Kaiser PK, Singh RP, Srivastava SK. Intrasurgical dynamics of macular hole surgery: an assessment of surgery-induced ultrastructural alterations with intraoperative optical coherence tomography. Retina. $2014 \mathrm{Feb} ; 34(2): 213-21$.

23 Forsaa VA, Krohn J. Air tamponade combined with nonsupine positioning in macular hole surgery for pseudophakic eyes. Retina. 2017 Sep;37(9):1750-6.

24 Ullrich S, Haritoglou C, Gass C, Schaumberger M, Ulbig MW, Kampik A. Macular hole size as a prognostic factor in macular hole surgery. Br J Ophthalmol. 2002 Apr;86(4):390-3.

25 Shukla SY, Afshar AR, Kiernan DF, Hariprasad SM. Outcomes of chronic macular hole surgical repair. Indian J Ophthalmol. $2014 \mathrm{Jul}$; 62(7):795-8. 
26 Michalewska Z, Michalewski J, DulczewskaCichecka K, Adelman RA, Nawrocki J. Temporal inverted internal limiting membrane flap technique versus classic inverted internal limiting membrane flap technique: a comparative study. Retina. 2015 Sep;35(9):1844-50.

27 Shiode Y, Morizane Y, Matoba R, Hirano M, Doi S, Toshima S, et al. The role of inverted internal limiting membrane flap in macular hole closure. Invest Ophthalmol Vis Sci. 2017 Sep 1;58(11):4847-55.

28 Kang SW, Ahn K, Ham DI. Types of macular hole closure and their clinical implications. $\mathrm{Br}$ J Ophthalmol. 2003 Aug;87(8):1015-9.

29 Morizane Y, Shiraga F, Kimura S, Hosokawa M, Shiode Y, Kawata T, et al. Autologous transplantation of the internal limiting membrane for refractory macular holes. Am J Ophthalmol. 2014 Apr;157(4):861-9.e1.
30 Bonińska K, Nawrocki J, Michalewska Z. Mechanism of "flap closure" after the inverted internal limiting membrane flap technique. Retina. 2018 Nov;38(11):2184-9.

31 Michalewska Z, Michalewski J, Cisiecki S, Adelman R, Nawrocki J. Correlation between foveal structure and visual outcome following macular hole surgery: a spectral optical coherence tomography study. Graefes Arch Clin Exp Ophthalmol. 2008 Jun;246(6):823-30.

32 Shen Y, Lin X, Zhang L, Wu M. Comparative efficacy evaluation of inverted internal limiting membrane flap technique and internal limiting membrane peeling in large macular holes: a systematic review and meta-analysis. BMC Ophthalmol. 2020 Jan 8;20(1):14.

33 Ooto S, Hangai M, Takayama K, Ueda-Arakawa N, Hanebuchi M, Yoshimura N. Photoreceptor damage and foveal sensitivity in surgi- cally closed macular holes: an adaptive optics scanning laser ophthalmoscopy study. Am J Ophthalmol. 2012 Jul;154(1):174-86.e2.

34 Ota H, Kunikata H, Aizawa N, Nakazawa T. Surgical results of internal limiting membrane flap inversion and internal limiting membrane peeling for macular hole. PLoS One. 2018;13(9):e0203789.

35 Chen SN. Large semicircular inverted internal limiting membrane flap in the treatment of macular hole in high myopia. Graefes Arch Clin Exp Ophthalmol. 2017 Dec;255(12): 2337-45.

36 Kuriyama S, Hayashi H, Jingami Y, Kuramoto N, Akita J, Matsumoto M. Efficacy of inverted internal limiting membrane flap technique for the treatment of macular hole in high myopia. Am J Ophthalmol. 2013 Jul;156(1):12531.e1. 